\title{
Bifurcations of Equilibrium Forms of NEMS/MEMS as a Sensor of Microphysical Alterations
}

\author{
Dmitrii Skubov ${ }^{1,2 a}$, Alexey Lukin ${ }^{2}$, Ivan Popov ${ }^{2}$ and Lev Shtukin ${ }^{1,2}$ \\ ${ }^{1}$ Institute of the Problems of mechanical engineers IPME RAS, 199178 St.-Petersburg Russia \\ ${ }^{2}$ St.-Petersburg Polytechnic University SPbPU, 195251, St.-Petersburg, Russia
}

\begin{abstract}
In our work the results of analytical and numerical investigation of equilibrium forms of round conductive and ferromagnetic micro-membrane situated in uniform electrostatic or magnetic static field and also the branching of equilibrium forms of the system of two and three nano-layers with direct currents are described. The structure of this type is practically realizable and exercisable as a sensor of alteration of external and inner mechanical and electrical characteristics. Exactly in a case of nano/micro schemes it is necessary to take in account the nonlinearity of its interaction, which leads to significantly nonlinear effects. On a base of nonlinearity we can determine the alteration of mechanical and electrical characteristics. This type of nonlinear effects mostly unattainable in macroscale but in case of nano- or microscale their realization gets off on the first plan and appears as a sensor of small physical alteration.
\end{abstract}

\section{Introduction}

In recent time the development and achievement of micro- and nanoelectromechanical systems (MEMS and NEMS) appeal the great interest of physics, biologists, electricians. The use of these devices is connected with their high sensitivity for nano- and micro-scale alterations of physical and biological parameters [1-6]. For example, it's used as a determination of molecular mass, quantum states, biochemical reactions, production of some new molecules and others nanoparticles, and also their sensitivity for alteration of different types of external microphysical magnitudes. With help of miniature nano- and micro-sensors the supersensitive for extremely small forces and masses have been found: for forces of the order some zepto-newton $\left(1 \mathrm{zN}=10^{-21} \mathrm{~N}\right)$, for masses - some zepto-gram. Reaching of this sensitivity is connected with attainment of Mega and even Giga-Hertz diapason of eigen frequency of their dynamical characteristics. Increasing of eigen frequency until $1 \mathrm{GHz}$ gives the opportunity to avoid the influence of some physical noise on microsensors, such as, for example, a temperature and humid fluctuations. So silicon material with length $1 \mu \mathrm{m}$, width $0.12 \mu \mathrm{m}$ and thickness $0.075 \mu \mathrm{m}$ has eigen frequency $1.03 \mathrm{GHz}$ with quality of order $Q \sim 10^{4}[3,4,12]$.

The important significance of the nano- and microactuators has been revealed $[1,2]$ as micro-switches with forward or rotary movement. These devices may be membranes or cantilevers and other types, also a high speed rotational actuator - contactless micro-gyroscope. It is necessary to say that not less important the microsensors based on «pull-in» effect have been obtained.

\footnotetext{
$\bar{a}$ Corresponding author: skubov.dsk@yandex.ru
}

This effect gives the opportunity to determine the microalteration of external or inner excitation [1]. «Pull-in» instability is an inherently nonlinear effect connected with disappearance of equilibrium forms of elastic part of sensitive and actuating mechanism. This effect occurs at achievement of electro or magneto static actions the determined critical value.

The great interest also is presented by the determination of multitude of equilibrium forms of elastic bodies, which are possible in MEMS/NEMS, their bifurcation and stability. The same mathematical task arises at the modeling of electromechanical devices including the ferromagnetic parts: cores and armatures of electromagnets, magnetically controlled materials (ferrite elastics) [10] and others.

In our work the results of analytical and numerical investigation of equilibrium forms of round conductive and ferromagnetic micro-membrane situated in uniform electrostatic or magnetic static field and also the branching of equilibrium forms of the system of two and three nano-layers (wires) with direct currents are described. The structure of this type is practically realizable [10] and exercisable as a sensor of alteration of external and inner mechanical and electrical characteristics. For example, it's one of the types of nano-resonators, used for measuring a mass of adhesing nanoparticle $[11,12]$. In this case the measuring of a mass of nanoparticle is realized based on the alteration of an eigen frequency of flexural oscillations of nanolayer.

It's important to note that exactly in a case of nano/micro schemes it is necessary to take in account the nonlinearity of interaction in them, which leads to 
significantly nonlinear effects. Namely on a base of nonlinearity we can determine the alteration of mechanical and electrical characteristics. This type of nonlinear effects mostly unattainable in macro scale but in case of nano- or microscale their realization gets off on the first plan and appears as a sensor of small physical alteration.

The change of mechanical and electrical characteristics of one of micro-layers MEMS/NEMS can bring to asymmetry in a form of bending flexure that is completely revealed with modern measuring equipment. With gradual change of magnetic induction in a case of ferromagnetic membrane deformation and the rise of current value in a case of wire deflection we again can obtain their symmetric, thereby determinate happened alteration in nano- or micro scales.

\section{Branching of equilibrium forms of ferromagnetic membrane}

\subsection{Case of one magnet}

The combined system of equations of linear elastic and nonlinear magneto-elastic tasks consists in equations of task of stationary distribution of magnetic field and the equation of elasticity of deformed body [19]. The ponderomotive forces are included in equations of deformation, which in technical applications should be related for undeformed body. For nearly situated sufficiently smooth surfaces of ferromagnetic bodies the surface forces are acted. The surface density $\boldsymbol{q}$ of which can be founded from expression $\frac{1}{2} H^{2} \times \nabla \mu$ passaged to the limit, then we have a discontinuity of magnetic penetrability $\mu$. With accuracy relatively of highest degree terms of $\frac{\mu_{0}}{\mu}$ the obtained expression for surface force, acting on ferromagnetic, have a next form:

$$
q=\frac{1}{2} \mu_{0} H_{0}^{2} n=\frac{1}{2}\left[\frac{\varphi(M)-\varphi \cdot(M)}{h(M)-u(M)}\right]^{2} n
$$

Here $\varphi$ и $\varphi_{\%}$ - the scalar magnetic potentials of the close-fitting surfaces of ferromagnetic, $h(M)$ and $u(M)$ - initial distance between surfaces and displacement in a normal arise from their elastic deformation. The largest simplification is achieved for ideal ferromagnetic bodies (when in ferromagnetic we can assume, that $\mu=\infty$ ). Therefore the value of magnetic potentials $\varphi$ and $\varphi_{s}$ will be the same in all points of surfaces $S$ and $S_{*}$. For the systems with nearly situated smooth surfaces with small flexure, as in a considerable case, the difference of potentials is equal to total current $I$, which is covered by power line of magnetic field, taken with corresponding sign. It is necessary to say that in this statement we neglect the boundary-layer effect, believing that the ratio of gap size to radius of membrane is very small.

The analogy propositions can be supposed believing that ferromagnetic membrane is situated nearly the pole of electromagnet. In this case the membrane may be considered as another pole. The same mathematical problem arises at investigation of equilibrium forms of conductive membrane in electrostatic field.

Thus we obtain the nonlinear boundary problem for determination the forms of membrane flexure with surface normal force, which itself depends on its deformation. The equilibrium equation and boundary conditions have a form:

$$
\Delta u+\frac{1}{2} \frac{\mu_{0} I^{2}}{T} \frac{1}{(h-u)^{2}}=0,\left.\quad u\right|_{\Gamma}=0
$$

Here $u$-displacement, $T$ - tension in unit of distance, $h$ - gap between a pole of electromagnet and undeformed surface of a membrane, $\Gamma-$ contour of membrane. The further investigation is realized for round membrane. Having entered the dimensionless displacement $v=\frac{u}{h}$ and dimensionless parameter $\kappa^{2}=\frac{1}{2} \frac{\mu_{0} I^{2}}{\pi h^{3}}$ we obtain the equation:

$$
\frac{\partial^{2} v}{\partial r^{2}}+\frac{1}{r} \frac{\partial v}{\partial r}+\frac{1}{r^{2}} \frac{\partial^{2} v}{\partial \theta^{2}}+\frac{\kappa^{2}}{(1-v)^{2}}=0
$$

Transforming to new unknown variable $w=1-v$ yields the equation:

$$
\frac{\partial^{2} v}{\partial r^{2}}+\frac{1}{r} \frac{\partial v}{\partial r}+\frac{1}{r^{2}} \frac{\partial^{2} v}{\partial v^{2}}-\frac{\kappa^{2}}{w^{2}}=0
$$

Let's consider the axisymmetric case of deformation, believing that displacement does not depend on $\theta$. The equation (4) with dimensionless argument $\rho=r / R$ takes a form:

$$
\frac{\partial^{2} v}{\partial \rho^{2}}+\frac{1}{\rho} \frac{\partial v}{\partial \rho}-\frac{\alpha^{2}}{w^{2}}=0 .
$$

where $R$ - radius of membrane $\alpha^{2}=\kappa^{2} R^{2}$ and boundary conditions:

$$
w^{\prime}(0)=0, \quad w(1)=1 .
$$

The numerical solution of boundary task (5),(6) is obtained with MATLAB program bvp4 (solver of "Boundary Value Problem for ordinary differential equations") [20].

The numerically obtained equilibrium forms at different values of parameter $\alpha$ are shown in Fig.1. It is important to note, that for some fixed value $\alpha$, which characterizes the induction of external magnetic field, more than one equilibrium states of membrane are corresponded.

The equilibrium forms by solid and dotted lines with the same color, obtained at different angle of inclination of the membrane surface on its edge are shown in Fig. 1.

The method of direct numerical solution described above doesn't perfectly satisfy a task of finding the complete quantity and stability of the equilibrium forms, corresponding to given value of parameter $\alpha$. For achievement the complete solution of the described boundary task we can use a method of building a phase portrait, proposed in [19]. 


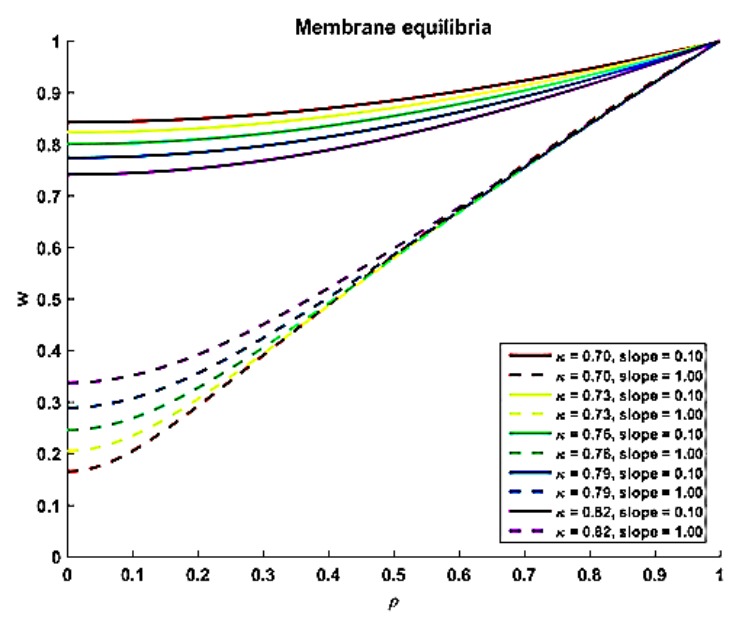

Figure 1. Equilibrium forms of ferromagnetic membrane

Using dimensionless radial coordinate $\tilde{\rho}=\alpha r$, we can transform the equilibrium equation in axisymmetric case (5) to the form:

$$
\frac{d^{2} w}{d \tilde{\rho}}+\frac{1}{\tilde{\rho}} \frac{d w}{d \tilde{\rho}}-\frac{1}{w^{2}}=0
$$

with boundary conditions:

$$
w^{\prime}(0)=0, \quad w(\alpha)=1 \text {. }
$$

The equation of the boundary problem (8) belongs to Emden-Fowler equations [16]

$$
\frac{d}{d \tilde{\rho}}\left(\tilde{\rho}^{k} \frac{d w}{d \tilde{\rho}}\right)-\tilde{\rho}^{m} w^{n}=0
$$

in our case $k=m=1, n=-2$. This equation is integrated in quadrature only at some values of $k, m$ other than zero. But it can be reduced to a system having excluded the argument $\tilde{\rho}$, that simplifies investigation. Let's input the new variables: argument $\tau$ and unknown function $\eta$ with relations $\tilde{\rho}=a e^{-\beta \tau}, w=b \eta e^{-\tau}$. For equation (8) we have obtained $a=\kappa, \beta=\frac{3}{2}, b=\sqrt[3]{\frac{9}{4} \kappa^{2}}$. It's important to note, that in view of boundary conditions $\eta(0)=\frac{1}{b}$, consequently at $a>\frac{2}{3} b>1$ and $\eta(0)<1$, and on the contrary at $a<\frac{2}{3}$ $b<1$ and $\eta(0)>1$. Transformed to function $\eta(\tau)$, we have obtained the next boundary problem:

$$
\eta^{\prime \prime}-2 \eta^{\prime}+\eta-\frac{1}{\eta^{2}}=0, \quad \eta(0)=\frac{1}{b}
$$

For this boundary task it is necessary to add the condition of limitation of displacement in a center, that is corresponded to limitation of $\lim _{\tau \rightarrow \infty} \eta(\tau) e^{-\tau}$. The autonomous equation of a boundary problem (11) can be rearranged as a system:

$$
\eta^{r}=v, \quad \theta^{r}=2 \theta-\eta+\frac{1}{\eta^{2}}
$$

On the phase plane for system (12) the critical point $\vartheta=0, \eta=1$ is an unstable focus. The derivative has a view:

$$
\frac{d v}{d \eta}=2-\frac{\eta}{v}+\frac{1}{v \eta^{2}}
$$

Thus the integral curve proceeding from focus, the part of which is corresponded to boundary task (11), at $\eta \rightarrow \infty$ asymptotically approaches to a straight $\theta=\eta$, remained below. The phase curve corresponding to boundary task is shown in Fig.2.

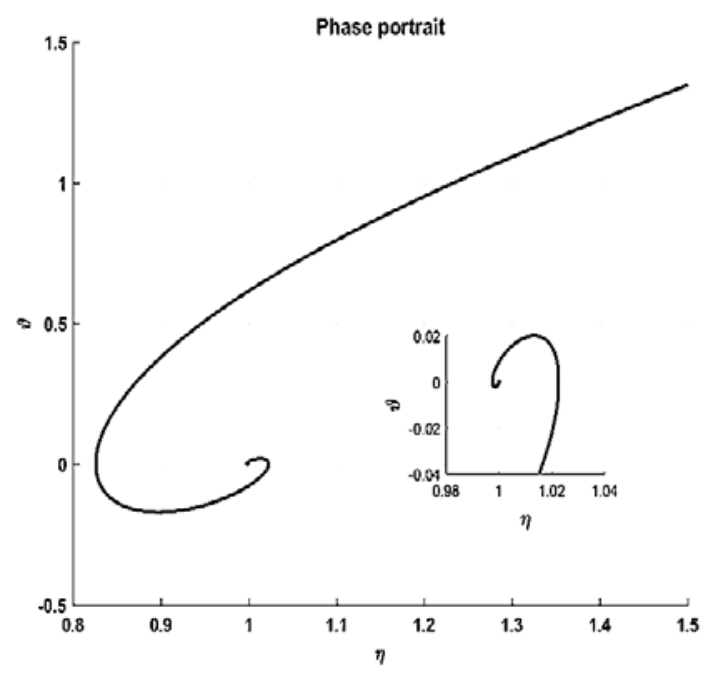

Figure 2. Phase curve corresponding to boundary task

On the appropriated integral curve

$$
\frac{d w}{d \tilde{\rho}}=\frac{1}{\eta(0)} \frac{2}{3 \alpha}(\eta-\vartheta) e^{\frac{1}{2} \tau}
$$

That corresponds to minimum of variable $w=1-v$, i.e. maximum of flexure in a center of membrane at it monotonically decreasing of flexure until zero on a boundary of membrane.

For found solution at prescribed value of parameter $\kappa$ and, consequently $b=\sqrt[3]{\frac{9}{4} x^{2}}$, it's necessary to take a vertical $\eta(0)=\frac{1}{b}$ on a phase plane of Fig.2. From the point of its crossing with integral curve, proceeding from the focus, and further along the corresponding phase trajectory segment, we have obtained the solution of axisymmetric boundary task. These crossings can be more than one, and consequently we have some solutions of our boundary problem.

At $\kappa<\frac{2}{3}$ this vertical is situated from the right of vertical $\eta=1$ and from the left at $\kappa>\frac{2}{3}$. The exclusion is the equilibrium form corresponding $\kappa=\frac{2}{a}$ and, consequently $b=1=\eta(0)$. The solution of boundary task (11) in this case completely lies on the phase curve proceeding from the focus and asymptotically approaches to a bisecting line of the first quadrant of phase plane Fig.2. The equation of the flexure has a form $v(\tilde{\rho})=1-\left(\frac{a \not \partial}{2}\right)^{2 / 3}$. The membrane, accordingly bended, is similar to funnel with bevel in a center. This solution and close to it are considered as a formal not corresponding to initial assumption for smallness of curvature of deformed membrane.

The dependence of maximal flexure in a center of membrane $v_{\mathrm{m}}$ (or correspondingly zero of $\left.w_{m}=1-v_{m}\right)$ from the value of parameter $\kappa$ is presented in Fig.3. 
The building of bifurcation diagram gives the possibility to determine the number and a form of equilibrium axisymmetric forms of the membrane. The upper branch of diagram is responsible for stable equilibrium position.

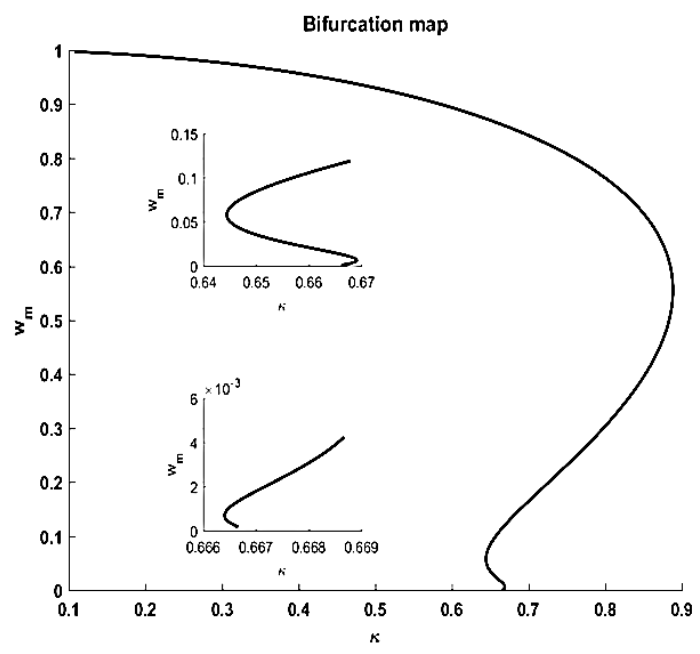

Figure 3. Diagram of branching of equilibrium positions

The found forms of equilibrium coincide with the results obtained by direct numerical solution of boundary problem (5),(6).

Owing to known theorem about non-existence of radial-asymmetrical solutions of nonlinear elliptic tasks in a ball of corresponding dimension (the round in our case) and at condition of nonnegative of external action $f(u)[7,8]$ the task of finding antisymmetric equilibrium forms doesn't have a solution. Our numerical and analytic results confirm the truth of this statement.

\subsection{Case of two magnets}

Let's consider a case of two magnets with identical poles facing to ferromagnetic membrane situated between them. Now the theorem [8] is unacceptable due to various signs of external ponderomotive action, which has a form

$$
f(u)=\frac{1}{2} \frac{\mu_{0} I^{2}}{T}\left[\frac{1}{(h-u)^{2}}-\frac{1}{(h+u)^{2}}\right]
$$

Thereby in this case it is possible the existence of antisymmetric forms of equilibrium position.

Let's turn to study the diagram of bifurcation of this boundary problem

$$
\Delta u+\frac{1}{2} \frac{\mu_{0} I^{2}}{T}\left[\frac{1}{(h-u)^{2}}-\frac{1}{(h+u)^{2}}\right]=0,\left.\quad u\right|_{\Gamma}=0
$$

Inputting to eq. (16) the dimensionless displacement $v=\frac{u}{h}$ and dimensionless parameter $\kappa^{2}=\frac{1}{2} \frac{\mu_{0} I^{2}}{T h^{3}}$ in analogy to previous case of one membrane we obtain the next equation for round membrane:

$$
\frac{\partial^{2} v}{\partial \rho^{2}}+\frac{1}{\rho} \frac{\partial v}{\partial \rho}+\frac{1}{\rho^{2}} \frac{\partial^{2} v}{\partial g^{2}}+\alpha^{2}\left[\frac{1}{(1-v)^{2}}-\frac{1}{(1+v)^{2}}\right]=0,
$$

were $\alpha=\kappa R$. This task has a trivial solution $v \equiv 0$ at any value of parameter $\alpha$. The points of bifurcation with appearance of nonzero solutions are determined at those parameters $\alpha$ at which the variation equation, built relatively to zero solution of (17), has nontrivial solution. This equation relatively a solution with $k$ harmonics on the angle coordinate with boundary homogenous condition has a form:

$$
\frac{d^{2} \xi_{k}}{d \rho^{2}}+\frac{1}{\rho} \frac{d \xi_{k}}{d \rho}+\left(4 \alpha^{2}-\frac{k^{2}}{\rho^{2}}\right) \xi_{k}=0
$$

Boundary conditions $\xi_{k}(1)=0$. This is a Bessel equation and its solution has a form $\xi_{k}=J_{k}(2 \alpha \rho)$, which in a sense of boundary problem has an eigenvalue at $2 \alpha=\lambda_{k i}, i=1,2, \ldots$, where $\lambda_{k i}$ - the roots of Bessel function of $k$ order $J_{k}\left(\lambda_{k i}\right)=0$. The first point of bifurcation corresponds to $k=0$ with axisymmetric form of deformation.

The task of direct finding of nontrivial forms of equilibrium states of the membrane (axisymmetric and antisymmetric) can be solved by numerical methods. For this aim the module of system MATLAB (Partial Differential Equations Toolbox), based on FEM [20] is applied. For definition of initial approximation for required solution at given value of parameter $\alpha$ we use a solution obtained at previous step of iteration procedure, obtained at less value $\alpha$. This method gives an opportunity for finding all the branches of bifurcation diagram. Acquirement of the points of branching $a=\frac{\lambda_{k i}}{2}$ obtained above analytically for different states of equilibrium essentially simplify this problem.

The symmetrical equilibrium forms of a membrane for $k=0$ at some values of parameter $\alpha$ are shown in Fig.5(a,b). These branches of forms correspond to points of bifurcation $\alpha=\frac{\lambda_{01}}{2}, \frac{\lambda_{02}}{2}$. The antisymmetric forms with $k=1$ corresponded to a branch issuant from $\alpha=\frac{\lambda_{11}}{2}, \frac{\lambda_{12}}{2}$ are shown in Fig.6(a,b). The corresponded diagram of branching is shown in Fig.7.

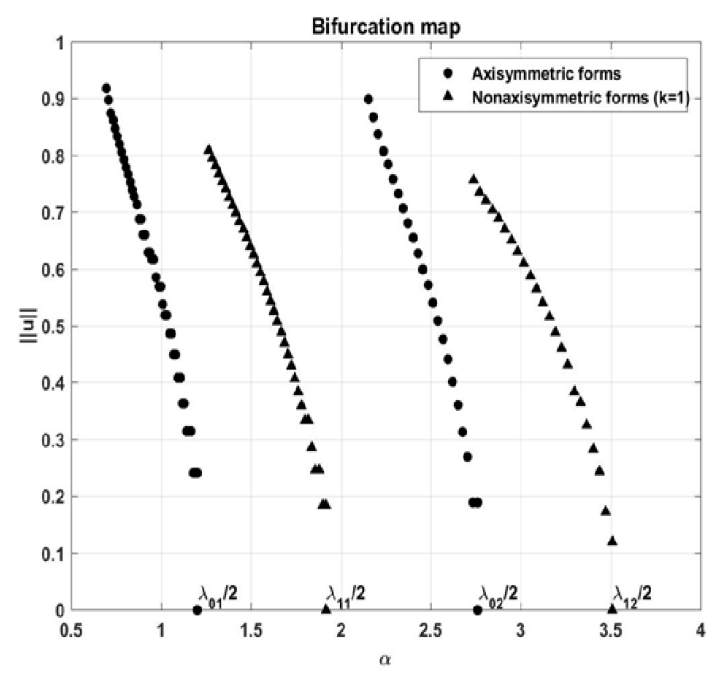

Figure 7. Bifurcation of the states of equilibrium 

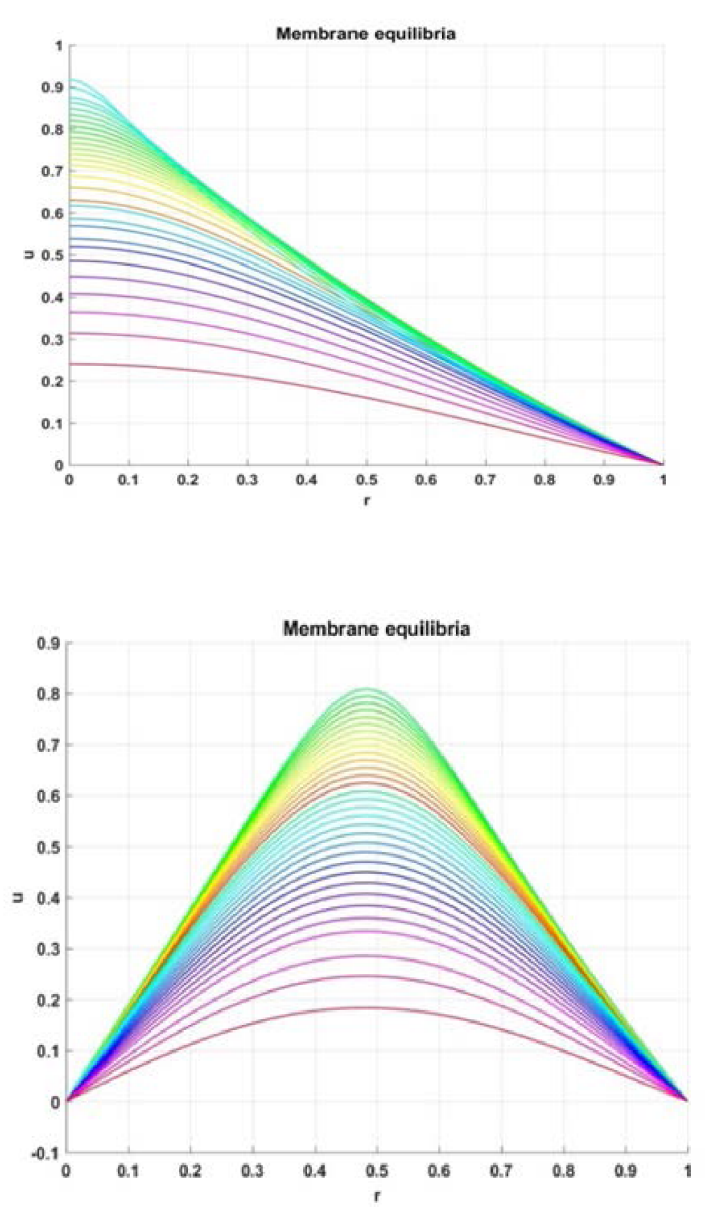

a)

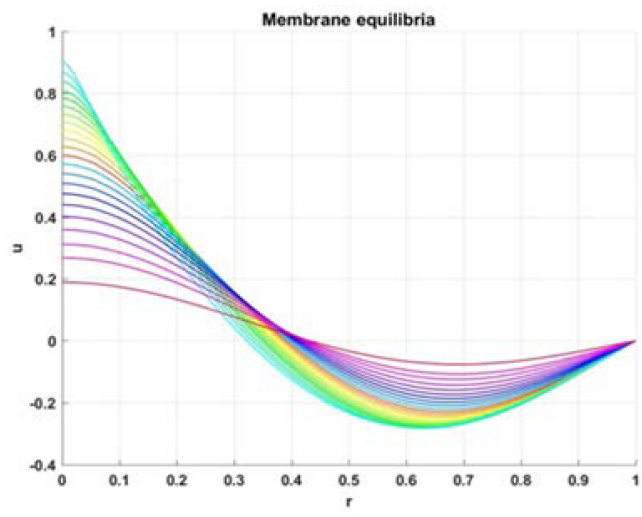

Figure 5. a,b

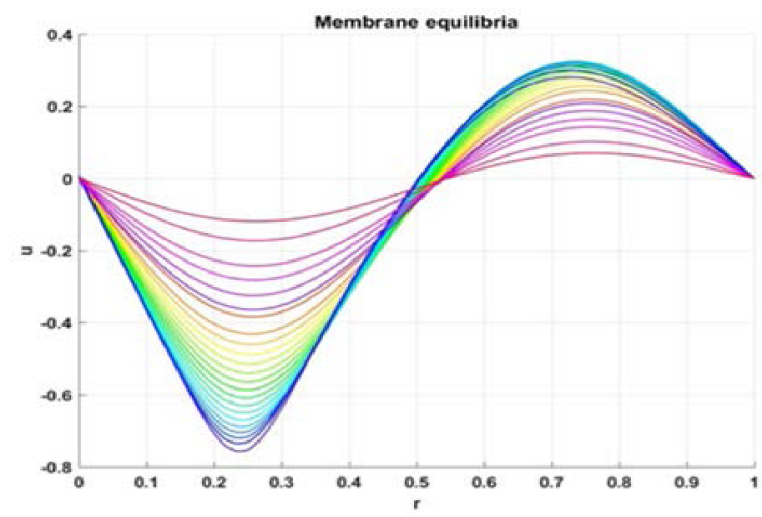

b)

Figure 6. a,b

It is interesting and is conform by the theory of branching that all nonzero forms are unstable. This result gives us the field of attraction of stable undeformed form of membrane. From this point of view it is important that the amplitude of nontrivial solution is increased at decreasing the value of parameter $\alpha$, proportional to quadrate of induction of external magnetic field.

\section{Branching of equilibriums of a system of nano-layers}

In this paragraph we can investigate the branching of equilibrium forms of three parallel micro- nanolayers MEMS and NEMS with constant currents (Fig.9). The system of this type is workable even in the nanoscale [2]. This micro/nanostructure can be used as a sensor of external and internal mechanical and electrical characteristics at their small change or as one of types of nanoresonators, which are used for measuring the mass of adhering nanoparticles [11,12]. In last case the measuring of a mass of nanoparticle is possible due to change in the eigen frequency of mechanical oscillations.
It is important to note that in a case of micro- nanocircuits the nonlinear interaction between them must be considered, which leads to nonlinear effects. On the base of nonlinear effects it is possible to identify changing the mechanical and electrical characteristics. The nonlinear effects of this type are mostly unattainable in macroscales, but in a case of nano- microscales their implementation is coming to the first plan and taking place as a sensor of small physical changes.

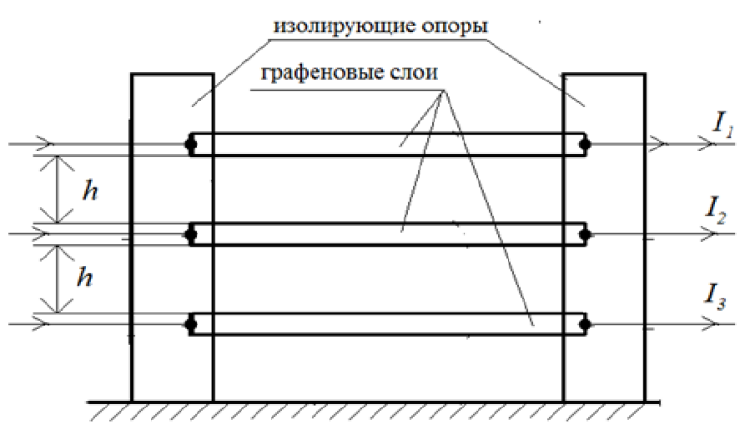

Figure 9. System of nanolayers

Changes in mechanical and electrical characteristics of one of the layers of MEMS/NEMS may cause 
asymmetry in the form of deflection, which is quite detectable by modern measuring equipment. With gradual change in a current in one of layers we can achieve their symmetry again, thereby identifying the changes in the micro/nanoscale.

Let's carry out the estimations of parameters of graphene nanostrings [5], at which their significant interaction is realized: the length of layer $l=1000 \mathrm{~nm}$, width $\delta=10 \mathrm{~nm}$, thickness $b=0.3 \mathrm{~nm}$. Thus, the resonance frequency of string oscillations reaches $30 \mathrm{MHz}$ with quality factor $Q \sim 100$. Using the expression for eigen frequency $\bar{\omega}$, we obtain the required tensile force

$$
T=\rho_{V} S \frac{l^{2} \omega^{2}}{\pi^{2}}=1,5 \cdot 10^{-12} \mathrm{~N}
$$

Using the expression for parameter $\lambda^{2}=\frac{\mu_{0} I^{2}}{2 \pi T h^{2}}$, entered below and characterizing the strong interaction of layers, and setting, that its value corresponds to a gap of the order $h \sim 10 \mathrm{~nm}$, we get the current value

$$
I_{0}=\frac{h \lambda}{l} \sqrt{\frac{\pi T}{\mu_{0}}}=40 \cdot 10^{-6} \mathrm{~A}=40 \mathrm{MkA},
$$

that is quite feasible in nanosystems.

In our work we study the static positions of the three parallel nano/microstrings with constant current and equal gaps between them, that in mathematical sense is close to consideration of equilibrium shapes of ferromagnetic membranes in a constant axisymmetric magnetic field [13].

Inputting the designation of currents in a case of their equality $I_{1}=I_{2}=I_{3}=I=$ const, we write the approximate expression for distributed attractive forces without regard to boundary effects

$$
q=\frac{\mu_{0} I^{2}}{2 \pi} \frac{r}{r^{2}}
$$

here $r$ - vector of distance between strings, which in a first approximation directs vertically to the strings [19]. The dynamical equation of a string with tension $T$, linear density $\rho$ and force of attraction $\boldsymbol{q}$ in a vector form:

$$
T u^{\prime \prime}-\rho \dot{u}+q=0
$$

The equations of equilibrium for three strings (without taking into account the interaction of extremes) are written in the form:

$$
\begin{aligned}
& T u_{1}^{H}+q_{12}=0, \\
& T u_{3}^{H}+q_{22}=0 .
\end{aligned}
$$

Here $q_{i j}$ - the forces of attraction from $j$ - th conductor with $i$-th, $u_{i}, i=1,2,3$ - displacements of the strings one by one due to their vertical deflection deformation, the derivative is taken along the length. The positive displacements of strings are directed vertically in the same direction as the positive direction of efforts. In the case of the attracting force difference currents are written in the form:

$$
\begin{array}{cc}
q_{12}=\frac{\mu_{0} I_{1} I_{2}}{2 \pi} \frac{1}{\left(h+u_{2}-u_{1}\right)^{\prime}}, & q_{21}=-q_{12} \\
q_{23}=\frac{\mu_{0} I_{2} I_{8}}{2 \pi} \frac{1}{\left(h+u_{8}-u_{2}\right)}, & q_{32}=-q_{23}
\end{array}
$$

here $h$ - initial gap between conductors (strings). Inputting the dimensionless coordinates and displacements $s=x / l, v_{i}=u_{i} / h$ and the main dimensionless physical parameter $\lambda^{2}=\frac{\mu_{a} I^{2} l^{2}}{2 \pi T h^{2}}$, where $I-$ a large scale of current value. Instead of the dimensional equations (21),(22) we will receive system of the equations in a dimensionless form:

$$
\begin{aligned}
v_{1}^{g}+\frac{\alpha_{1} \lambda^{2}}{1+v_{2}-v_{1}} & =0, \\
v_{2}^{n}-\frac{\alpha_{1} \lambda^{2}}{1+v_{2}-v_{1}}+\frac{\alpha_{2} \lambda^{2}}{1+v_{3}-v_{2}}=0, & \\
v_{3}^{r}-\frac{\alpha_{2} \lambda^{2}}{1+v_{3}-v_{2}} & =0,
\end{aligned}
$$

The sum of derivations of deflections owing to symmetry of deformation of strings on length is equal to zero. And, therefore, the sum of deflections, owing to zero regional conditions, is also equal to zero:

$$
v_{1}+v_{2}+v_{3}=0
$$

Let's enter the differences of deflections: $z_{1}=v_{1}-v_{2}, z_{2}=v_{2}-v_{3}$ and, subtracting the second equation from the first and third of the second, we will receive system of two nonlinear equations:

$$
\begin{aligned}
& z_{1}^{\prime \prime}+\frac{2 \alpha_{1} \lambda^{2}}{1-z_{1}}-\frac{\alpha_{2} \lambda^{2}}{1-z_{2}}=0 \\
& z_{2}^{\prime \prime}+\frac{2 \alpha_{2} \lambda^{2}}{1-z_{2}}-\frac{\alpha_{1} \lambda^{2}}{1-z_{1}}=0
\end{aligned}
$$

with zero boundary conditions. Deflections of each of strings, using the entered variables, are written in a form:

$$
v_{1}=\frac{2 z_{1}+z_{2}}{3}, \quad v_{2}=\frac{z_{2}-z_{1}}{3}, v_{3}=-\frac{2 z_{2}+z_{1}}{3} .
$$

Obtained boundary problem (25) at equality of currents allows the symmetric solution with not deformed average string and the identical opposite directed deflections of extremes $v_{2}=0, v_{1}=-v_{3}$ (extreme strings cave in towards), when $z_{1}=z_{2}=z_{3}$ and instead of two equations (25) we have one:

$$
z^{\prime \prime}+\frac{\lambda^{2}}{1-z}=0
$$

with boundary conditions: $z(0)=0, z(1)=0$. The boundary problem (27) realized in a case $\alpha_{1}=\alpha_{2}=1$ is completely equivalent to the problem of a deflection of two strings considered by us earlier with direct identical currents [19]. Its bifurcation diagram has two solution sat value of parameter $\lambda<\lambda_{*} \approx 1.54$ with a smaller steady and bigger unstable symmetric form of a deflection. Rewriting the equations (25) in a form:

$$
z_{1}^{\prime \prime}+2 z_{2}^{\prime \prime}+\frac{3 \lambda^{2}}{1-z_{2}}=0,2 z_{1}^{\prime \prime}+z_{2}^{\prime \prime}+\frac{3 \lambda^{2}}{1-z_{1}}=0
$$


and multiplying the first equation on $z_{2}^{r}$ and second on $z_{1}^{r}$, we have obtained after summation the integral of energy:

$$
\left(z_{1}^{s}\right)^{2}+\left(z_{2}^{\prime}\right)^{2}+z_{1}^{s} z_{2}^{r}-3 \lambda^{2} \ln \left(1-z_{1}\right)-3 \lambda^{2} \ln \left(1-z_{2}\right)=
$$$$
h=\text { const, }
$$

having the symmetry relatively $z_{1}, z_{2}$. If to look for the private symmetric solution $z_{1}=z_{2}=z$, the integral is given to a form:

$$
\left(z^{\prime}\right)^{2}-2 \lambda^{2} \ln (1-z)=h=\text { const },
$$

what corresponds to a boundary task for two strings.

The solution of a boundary task (25) is passed numerically, using the subprogramme bvp4c MatLab, and Galerkin's method. The numerically obtained symmetric and asymmetric relatively average layer equilibrium states, corresponding to various value of $\lambda$ are shown in Fig.12,13

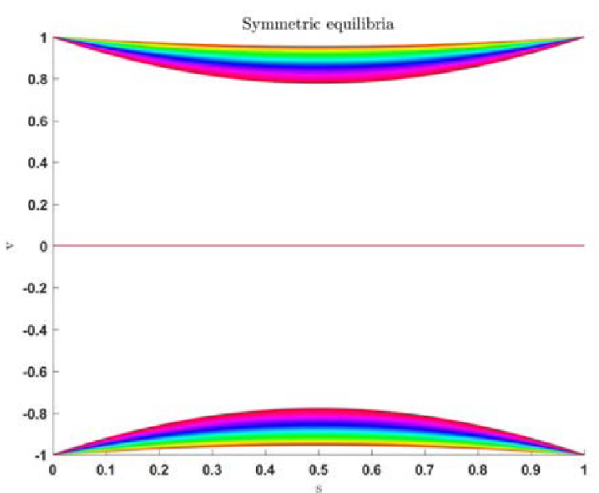

Figure 12. Symmetric equilibrium states

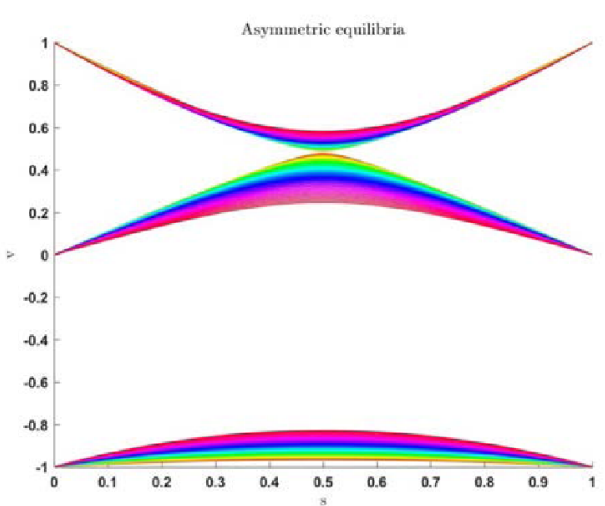

Figure 13. Asymmetric equilibrium states

Projective conditions on Galerkin in case of forms of a deflection, symmetric concerning the middle, are received at taking of the approximate solution at a form:

$$
z_{i}=x_{i} \sin \pi s, \quad i=1,2 .
$$

Projective conditions of system (28) for the taken forms of deflections (31) have a form:

$$
\begin{aligned}
& -\pi^{2}\left[2 x_{1}+x_{2}\right] \int_{a_{1}}^{1} \sin ^{2} \pi s d s+3 a_{1} \lambda^{2} \int_{0_{1}^{1}}^{1} \frac{\sin \pi s}{1-x_{1} \sin \pi s} d s=0 \\
& -\pi^{2}\left[2 x_{2}+x_{1}\right] \int_{0}^{1} \sin ^{2} \pi s d s+3 a_{2} \lambda^{2} \int_{0}^{1-x_{2} \sin \pi s} d s=0 .
\end{aligned}
$$

In result the system (32) takes a form:

$$
\begin{aligned}
& \frac{\pi^{2}}{2}\left[2 x_{1}+x_{2}\right]-\frac{3 \alpha_{1} \lambda^{2}}{\pi x_{1}}\left[\pi\left(\frac{1}{\sqrt{1-x_{1}^{2}}}-1\right)+\frac{2 \arcsin x_{1}}{\sqrt{1-x_{1}^{2}}}\right]=0, \\
& \frac{\pi^{2}}{2}\left[2 x_{2}+x_{1}\right]-\frac{3 \alpha_{2} \lambda^{2}}{\pi x_{2}}\left[\pi\left(\frac{1}{\sqrt{1-x_{2}^{2}}}-1\right)+\frac{2 \arcsin x_{2}}{\sqrt{1-x_{2}^{2}}}\right]=0 .
\end{aligned}
$$

The bifurcation diagram of solutions of a system (33) in a case of equal currents represents in Fig.14. By one color and one type of a marker in Fig. 14 the equilibrium states of three layers which are realized in common are designated in one color.

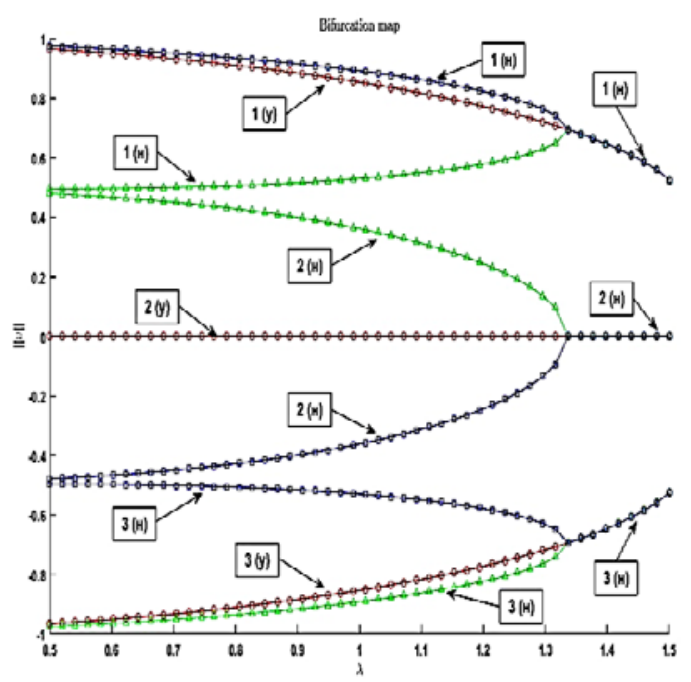

Figure 14. Bifurcation diagram in a case of identical currents: $\alpha_{1}=\alpha_{2}=1$

The branching of forms of balance of three strings in a case of identical currents happens in the form of a threefold point with steady until value of parameter $\lambda_{*} \approx 1.34$ neutral zero position of the second (average) string. At the value of parameter $\lambda$, exceeding $\lambda$, the neutral position of a middle string became unstable and asymmetric forms, at which the average string is deformed, are vanished. At achievement of parameter $\lambda$ the value 1.53 all equilibrium positions are disappeared, that physically corresponds to adhesion of strings (violation of the conditions of the studied model).

Transition through a symmetric case of identical currents is of special interest, at gradual (quasistationary) increasing of current in one of extreme strings. At the same time two boundary problems are solved. The first corresponds to smaller current in one of extreme strings, and identical in two others $\left(\alpha_{1}<1, \alpha_{2}=1\right)$ the second to bigger current in extreme, than in two others $\left(\alpha_{1}>1, \alpha_{2}=1\right)$.

As entry conditions of the numerical solution of a boundary task the solution of the linearized system (25) was made. The linearized system at small deflections has the following appearance: 


$$
\begin{aligned}
& \xi_{1}^{I I}+2 \lambda^{2}\left(1+\xi_{1}\right)-\lambda^{2}\left(1+\xi_{2}\right)=0 \\
& \xi_{2}^{I I}+2 \lambda^{2}\left(1+\xi_{2}\right)-\lambda^{2}\left(1+\xi_{1}\right)=0 \\
& \text { Or } \\
& \xi_{1}^{I I}+\lambda^{2}\left(2+2 \xi_{1}-\xi_{2}\right)=-\lambda^{2} \\
& \qquad \xi_{2}^{I I}+\lambda^{2}\left(2+2 \xi_{2}-\xi_{1}\right)=-\lambda^{2} .
\end{aligned}
$$

The system (34) at zero boundary conditions, what, in fact, means equality $\xi_{1}=\xi_{2}$, takes a form of one equation:

$$
\xi^{H}+\lambda^{2} \xi=-\lambda^{2}
$$

Under zero boundary conditions the boundary task (35) has the solution:

$$
\xi=-1+\cos \lambda s+(1-\cos \lambda) \frac{\sin \lambda s}{\sin \lambda},
$$

Linear approximation (36) suits for system (25) only at small currents - small $\lambda$ and corresponds only to small deflections.

For search of points of branching it is necessary to investigate compliance of eigenvalues of the equations in variations concerning the found symmetric form with value of parameter $\lambda[14,15]$. Proceeding from the numerical solution of an uniform nonlinear boundary task (25), branching happens at values $\lambda=\lambda_{*} \approx 1.34$ This value coincides with value $\lambda=\lambda$, at which there is a bifurcation of symmetric forms of equilibrium "contact" of static forms of middle string with one of extreme strings to further loss of the symmetric solution.

The variation equation for system (25) in case of the symmetric solution at $\lambda=\lambda_{0}, z=z_{0}(s)$ is written in a form:

$\xi^{\prime \prime}+\frac{\lambda^{2}}{\left(1-z_{0}(s)\right)^{2}} \xi=0$

with uniform boundary conditions:

$$
\xi(0)=\xi(1)=0, \eta(0)=\eta(1)=0 .
$$

The equation (37) with zero regional conditions is a linear boundary task with variable coefficient. Due to positivity of the variable coefficient in a differential equation (37) $\left(0<z_{0}(s)<1\right)$ the eigenvalue of variation task (25) decreases. The second item plays a role of the negative distributed modulus of foundation. The point of branching corresponds to coincidence of eigenvalue of a variation boundary task (37) with own value of parameter $\lambda$. Stability of asymmetrical forms is defined by calculation of eigen frequencies of the dynamic uniform tasks received by linearization of a system (25) concerning the asymmetrical nonlinear deflections [14].

$$
\begin{aligned}
& \xi^{\prime \prime}+\frac{2 \alpha_{1} \lambda^{2}}{\left(1-z_{10}\right)^{2}} \xi+\frac{\alpha_{2} \lambda^{2}}{\left(1-z_{20}\right)^{2}} \eta-\rho \ddot{\xi}=0 \\
& \eta^{\prime \prime}+\frac{2 \alpha_{2} \lambda^{2}}{\left(1-z_{20}\right)^{2}} \eta+\frac{\alpha_{1} \lambda^{2}}{\left(1-z_{10}\right)^{2}} \xi-\rho \ddot{\eta}=0 .
\end{aligned}
$$

Points of branching correspond to the equality to zero the eigen frequencies of linearized dynamic boundary task (38).
Important achievement of the real work is application of the theory of branching to new type nano - micro electromechanical schemes with possible using of the received structure of branching of the equilibrium forms for definition its nano- micro scale physical changes.

\section{Acknowledgements}

Work is performed with assistance of a grant of the RFBR 14-01-00845.

\section{References}

1. Wen-Ming Zhang, Han Yan, Zhi-Ke Peng, Guang Meng, Electrostatic pull-in instability in MEMS/NEMS: A review - Sensors and Actuators A 214 p.187-218 (2014)

2. Eom K., Harold S. Park (et al) Nanomechanical resonators and their applications in biological/chemical detection: Nanomechanics principles. Physics Reports, V. 503 p. 115 - 163 (2011)

3. Scott Bunch J. (et al) Electromechanical Resonators from Graphene Sheets V. 315 p. 490 - 493 (2007)

4. Chen C. (et al) Performance of monolayer graphene, Nature Nanotechnology, V. 4 p. 861-867 (2009)

5. He X.Q., Kitipornchai S., Liew K.M. Resonance analysis of multi-layered graphene sheets used as nanoscale resonators, Nanotechnology V.16 p. 2086 - 2091 (2009)

6. John A. Pelesko, Tobin A. Driscoll The effect of the small-aspect-ratio approximation on canonical electrostatic MEMS models Journal of Engineering Mathematics, 53, p. 239-25 (2005)

7. B. Gidas, Wei-Ming Ni, L. N. Nirenberg Symmetry and Related Properties via the Maximum Principle Commun. Math. Phys. 68 p.209-243 (1979)

8. Dong Ye., Feng Zhou On a general family of nonautonomous elliptic and parabolic equations Calc. Var. 37 p.259-274 (2010)

9. Griberg Ya.S. (et al) Nanomechanical resonators, Sucs.of Phys.Sience, 4 V.182 p.407 - 436 (2012)

10. Raiher Yu.L., Stolbov O.V., Stepanov G.V. Deformation of a round membrane (ferro-elastic) in uniform magnetic field, Journ. Technic. Phys. V.78, issue 9, p.69-76 (2008)

11. Morozov N.F., Berinsrii I.E., Indeitsev D.A., Privalova O.V., Skubov D.Yu., Shtukin L.V. Failure of oscillations of graphene resonator as a method of determination of its spectral characteristics, DAS, V. 456, № 5, p.537 - 540 (2014)

12. Shtukin L.V., Berinskii I.E., Indeitsev D.A., Morozov N.F., Skubov D.Yu. Electromechanical models of nanoresonators, Physic. Mezomechanics, V. 19, № 1 (2016)

13. Skubov D.Yu., Lukin A.V., Popov I.A., Privalova O.V., Shtukin L.V. Branching of equlibrium forms of nonlinear NEMS/MEMS Proc. of III Intern. School-NDM, p. 274-281 (2016) 
14. Vainberg M.M., Trenogin V.A. The theory of bifurcation of nonlinear equations - M.: Science, (1969)

15. Antman St., Keller J. B. Bifurcation theory and nonlinear eigenvalue problems - M.: World, (1974)

16. Sansone J. Ordinary differential equations - M.: In.Lit., (1953)

17. Bellman R. Theory of stability solutions of differential equations - M.: In.Lit. (1954)

18. Fridman V.M. Theory of elastic oscillations. Equations and methods - St.Petersb.: Sciece (2014)

19. Skubov D.Yu., Khodzhaev K.Sh. Nonlinear electromechanics -Springer-Verlag (2003)

20. Matlab Documentation, The MathWorks, Inc., 2015 